\title{
REGISTER RERUM ad Vol. 37
}

\section{9}

Confecit G. Boehm, Basel

Actinístía, v. Latímcría chalumnae

Action potentials, v. Nervenfasern

Adenohypophysis, v. Hypothalamus, Neurosecretory system

Afferente Nervenfasern, v. Nervenfasern

Anatomie des Menschen, normale, Lehrbuch (8. Ami.) (B) 192

Anatomie, v. Latímería chalumnae

Annual ovarian cycle, v. Ovarium

Antirhachitie substances, v. Epiphysial complex

Aorta, v. Carotid body

Aortic bodies, v. Carotid body

Argyrophile Basalzellen, v. Pflasterepithel

Arteria carotis, v. Carotid body

Arteria subodontalis, v. Dentes

Auge, v. Eye

Aves, v. Carotid body

Axons; experimental degeneration of optic axons in the lateral geniculate

body of the cat 281

«Barrera epitelial argentófila», v. Pflasterepithel

Basalzellen, v. Pflasterepithel

Base cranienne, v. Cranial synostosis

Biopsien, v. Pflasterepithel

Birds, v. Carotid body

Blood circulation in the rodent teeth of the rat

Blood circulation, v. Vascular supply

Blood pressure, v. Carotid body

Blood sinusoids, v. Carotid body

Blutdruckzügler, v. Carotid body

Body sections, primary, v. Körperabschnitte

Bone, v. Cranial synostosis, Ossification

Bulbus caroticus, v. Carotid body

Calcification, v. Ossification

Canal thoracique; anatomie du canal thoracique et des principaux collecteurs lymphatiques du tronc chez Thomme 20

Carotid body system of the higher vertebrates 265

Cat; experimental degeneration of optic axons in the lateral geniculate body of the cat 281

-; v. Felís domestica 
Catfish, indian, v. Heteropneustes

Cerebrum, v. Epiphysial complex, Geniculate body, Hypothalamus, Neurosecretory system, Thalamus

Chambehs, Robert (1881-1957). An appreciation of his scientific career . . 1

Chat, v. Felís domestica

Chemoreceptive system, v. Carotid body

(B) = Book reviews - Livres nouveaux - Buchbesprechungen

390

Register rerum

Cholinesterase, v. Muskelfasern

Chrome alum hematoxylin phloxin technique (Gomori), v. Hypothalamus,

Neurosecretory technique Coelacanthídae, v. Latimeria chalumnae Collecteurs lymphatiques, v.

Lymphatiques Coloration, v. Neurosecretory system Cordylus polyzonus, v. Lizards Corps

géniculé lateral, v. Geniculate body Corpus geniculatum laterale, v. Geniculate body Crane, v.

Cranial synostosis, Ossification

Cranial synostosis, premature, in man, pathogenesis 351

Cranium, v. Ossification

Crossopterygíi, v. Latimeria chalumnae

Cutis, v. Scrotalhaut

Cycle; annual ovarian cycle, v. Ovarium

Cysterna chyli, v. Canal thoracique

Cytochemistry, v. Hypothalamus, Muskelfasern, Neurosecretory system,

Pflasterepithel

Deformity, v. Cranial synostosis

"Degenerating boutons", v. Degeneration

Degeneration; experimental degeneration of optic axons in the lateral geni

culate body of the cat 281

$-;$ v. Nucleus anterior thalami

Dental lamina, v. Dentes

Dentes; blood circulation in the rodent teeth of the rat

-; les racines dentaires. Leur développement; leur résorption au niveau des dents temporaires 210

--; the vascular supply to the dental lamina during early development . . 232

Development of the interstitial cells in the testis of the albino rats .... 125

Development of the rat vagina 106

Development; the vascular supply to the dental lamina during early develop

ment 232

$-; \mathrm{v}$. Dentes

Diaphragma; das histologische Querschnittsbild des Rattenzwerchfells bei

Fixierung in verschiedenen Funktionszuständen 252

Diencephalon, v. Hypothalamus, Neurosecretory system

Differenzierungen der Scrotalhaut von Perodíctícus potto (MüUer 1766) im

Vergleich mit anderen Prosímíae

80

Difformité, v. Cranial synostosis

Ductus thoracicus, v. Canal thoracique

Efferente Nervenfasern, v. Nervenfasern 
Eidechsen, v. Lizards

Electron microscopic study of the foetal membranous ossification .... 370

Embryo; an electron microscopic study of the foetal membranous ossification 370

-; v. Cranial synostosis

Endplatten, motorische, v. Muskelfasern

Epiphysial complex; epithelial organization and histogenesis of the epiphysial complex in lizards 310

Register rerum

391

Epithel; Beiträge zur Kenntnis des geschichteten Pflasterepithels, Supple-

mentum 35 (=1 ad Vol. 37) Epithelial organization and histogenesis of the epiphysial complex in lizards 310 Excitation; synchronisation générale de la transmission de Гexcitation,

v. Nervenfasern Eye; "third eye", v. Lizards

Färbung, v. Neurosecretory system

Fasertypen im Muskel, v. Zwerchfell

Feinstruktur, v. Ossification, Pflasterepithel

«Felderstruktur» (Muskel), v. Zwerchfell

Felís domestica; Bau und funktionelle Bedeutung einiger isolierter markhal-

tiger Nervenfasern des Musculus piriformis der Katze 137

-; morphologische Beobachtungen im Hypothalamus der Katze nach

Unterbindung des Pankreasganges 193

-; experimental degeneration of the optic axons in the lateral geniculate

body of the cat 281

-; v. Dentes Ferments, v. Muskelfasern «Fibrillenstruktur» (Muskel), v. Zwerchfell Fixation, v. Neurosecretory system Fixation (mögliche Artefakte), v. Zwerchfell

Foetal membranous ossification, an electron microscopic study $\quad 370$

Formation de soutien, v. Latimería chalumnae

Frayer, v. Heteropneustes

Funktion (Funktionelle Beziehung, Funktionszustände), v. Nervenfasern,

Zwerchfell Fuseau neuro-musculaire, v. Nervenfasern

Galago crassícaudatus, v. Scrotalhaut

Ganglions lymphatiques, v. Canal thoraciques

Gefäße, v. Carotid body, Halsvenen, Rodent teeth, Vascular supply

Gefäße (Lymphgefäße), v. Canal thoracique

Geniculate body, lateral; experimental degeneration of optic axons in the

lateral geniculate body of the cat

281

Gewichte der Hauptkörperabschnitte, v. Körperabschnitte

Glande carotidienne, v. Carotid body

Glandes (peau du scrotum de certains prosimiens), v. Scrotalhaut

Glandulae cutis, v. Scrotalhaut

Glomus caroticum, v. Carotid body

«Goldkette» (nack Silberimprägnation), v. Pflasterepithel

Gomori chrome alum hematoxylin phloxin technique, v. Hypothalamus,

Neurosecretory system Gyrus cinguli, v. Nucleus anterior thalami

Halsvenen; Beitrag zum Studium von Varietäten der subkutanen Halsvenen

des Menschen 298 
Hauptkörperabschnitte, v. Körperabschnitte

Hautdrüsen (bei Prosímiae), v. Scrotalhaut

Hematoxylin; Gomori chrome alum hematoxylin phloxin technique, v. Hypothalamus,

Neurosecretory system

392

Register rerum

Herringkörper, v. Hypothalamus

Heteropneustes; a study on the correlation between the structure of the pitui

tary gland of the indian catfish Heteropneustes and the seasonal changes

in the ovary

47

Histochemistry, v. Hypothalamus, Muskelfasern, Neurosecretory system

Histogenesis, v. Epiphysial complex

Hormones, v. Pituitary gland

Hypophysis; a study on the correlation between the structure of the pitui

tary gland of the indian catfish Heteropneustes and the seasonal changes

in the ovary

47

-; v. Hypothalamus, Neurosecretory system

Hypothalamus; morphologische Beobachtungen im Hypothalamus der Katze

nach Unterbindung des Pankreasganges 193

-; v. Neurosecretory system

Incisivi, v. Dentes Indian catfish, v. Heteropneustes Innervation, v. Nervenfasern Internodien, v.

Nervenfasern

Interstitial cells in the testis of the albino rats, development 125

Jahreszeitliche Anderungen, v. Ovarium

Japaner; die Massenanteile der einzelnen Körperabschnitte der Japaner . . 385

Japonais, v. Japaner

Kaninchen, v. Racines dentaires

Katze; morphologische Beobachtungen im Hypothalamus der Katze nach

Unterbindung des Pankreasganges 193

$-;$ v. Felís domestica

Kniehöcker, seitlicher, v. Geniculate body

Knochen, v. Cranial synostosis, Ossification

Körperabschnitte; die Massenanteile der einzelnen Körperabschnitte der Ja

paner 385

Lacertilian epiphysis, v. Lizards

Laichen, v. Heteropneustes

Lamina, dental lamina, v. Dentes

Lapin, v. Racines dentaires

Lateral geniculate body, v. Geniculate body

Latimería chalumnae, anatomie (Tome I: Squelette, muscles et formation de soutien) (B) 280

Lehrbuch der normalen Anatomie des Menschen (8. Aufl.) (B)

192

Leye $^{3} / 8$ sche interstitielle Zellen, v. Interstitial cells

Lézards, v. Lizards

Lizards; epithelial organization and histogenesis of the epiphysial complex in

lizards 310 
Loris tardígradus, v. Scrotalhaut

Lorisoídea, v. Scrotalhaut

Lymphatiques, collecteurs lymphatiques; anatomie du canal thoracique et

des principaux collecteurs lymphatiques du tronc chez l'homme ... 20

Lymphgefäße, v. Lymphatiques

Register rerum

393

Mabuya sulcata, v. Lizards

Malformation, v. Cranial synostosis

Mammalia, v. Carotid body

Man, v. Anatomie, Canal thoracique, Halsvenen, Muskelfasern, Thalamus

Markhaltige Nervenfasern; Bau und funktionelle Bedeutung einiger isolierter markhaltiger

Nervenfasern des Musculus piriformis der Katze . 137

Massenanteile der einzelnen Körperabschnitte der Japaner 385

Membranous ossification, foetal, electron microscopic study

Methode, v. Neurosecretory system, Pflasterepithel

Mícrocßbus murinus, v. Scrotalhaut

Milk-teeth, v. Racines dentaires

Mißbildung, v. Cranial synostosis

Morphogenesis, v. Cranial synostosis

Motoric end-plates, v. Muskelfasern

Motor-units, v. Musculus piriformis

Mucosa, v. Pflasterepithel

Muscle spindles, $v$. Musculus piriformis

Muscles, v. Latimeria chalumnae, Musculus piriformis, Muskelfasern, Zwerchfell

Musculus piriformis; Bau und funktionelle Bedeutung einiger isolierter

markhaltiger Nervenfasern des Musculus piriformis der Katze . . . 137

Muskel; das histologische Querschnittsbild des Rattenzwerchfells bei Fixie-

rung in verschiedenen Funktionszuständen 252

-; v. Latimeria chalumnae, Musculus piriformis, Muskelfasern

Muskelfasern; Länge und Anordnung der Muskelfasern in menschlichen Ske-

letmuskeln 217

Muskelspindeln, v. Musculus piriformis

Necrologia

Nerven (Nervensystem, Zentralnervensystem), v. Carotid body, Degeneration, Epiphysial complex, Geniculate body, Hypothalamus, Nervenfasern, Neurosecretory system, Thalamus Nervenfasern; Bau und funktionelle Bedeutung einiger isolierter markhalti ger Nervenfasern des Musculus piriformis der Katze 137

Neurohypophysis, v. Hypothalamus, Neurosecretory system

Neurosecretory system; factors influencing the histological visualization of the neurosecretory system of the rat, when stained by Gomorí chrome

alum hematoxylin phloxin technique 153

Nodi lymphatici, v. Canal thoracique

Nucleus anterior thalami, zur Frage seiner Projektion

Nucleus infundibularis tuberis, v. Hypothalamus

Nucleus paraventricularis, v. Neurosecretory system 
Nucleus supraopticus, v. Hypothalamus, Neurosecretory system

Nyctícebus coucang, v. Scrotalhaut

Odontoblastes, v. Dentes Odontoclastes, v. Racines dentaires Odontogenetic regions, v. Vascular supply Oeil, v. Eye

Oesophagus, v. Pflasterepithel Oiseaux, v. Carotid body

394 Register rerum

Ontogenesis; development of the interstitial cells in the testis of the albino

rat 125

-; les racines dentaires. Leur développement; leur résorption au niveau des

dents temporaires 210

- ; development of the rat vagina 106

-; the vascular supply to the dental lamina during early development . . 232

Ontogenesis, v. Cranial synostosis, Epiphysial complex, Ossification

Optic axons; experimental degeneration of optic axons in the lateral geni-

culate body of the cat 281

Organum parietale, v. Epiphysial complex

Oryctolagus cunículus, v. Racines dentaires

Os, v. Cranial synostosis, Ossification

Ossification; an electron miscroscopic study of the foetal membranous ossi

fication $\quad 370$

Osteoblasts, v. Ossification

Ovarium; a study on the correlation between the structure of the pituitary

gland of the indian catfish Heteropneustes and the seasonal changes in

the ovary

47

Pankreasgang; morphologische Beobachtungen im Hypothalamus der Katze

nach Unterbindung des Pankreasganges

193

Paraganglion, v. Carotid body

Parietalauge, v. Epiphysial complex

Parietal organ, v, Epiphysial complex

Parties du corps, v. Körperabschnitte

Pathogenesis, v. Synostosis

Peau du scrotum, glandes, de certains prosimiens, v. Scrotalhaut

Perilaminar mesenchyme (dental lamina), v. Ontogenesis

Periodontium, v. Rodent teeth

Perodíctícus potto; Differenzierungen der Scrotalhaut von Perodictícus potto

(Müller 1766) im Vergleich mit anderen Prosímíae

80

Pflasterepithel, geschichtetes, Beiträge zu seiner Kenntnis, Supplementum 35 (=1 ad Vol. 37)

Phloxin; Gomori chrome alum hematoxylin phloxin technique, v. Hypothalamus, Neurosecretory system

Pineal organ, v. Epiphysial complex

Pisces, v. Latímería chalumnae

Pituitary gland; a study on the correlation between the structure of the pitui

tary gland of the indian catfish Heteropneustes and the seasonal changes

in the ovary

47

-; v. Hypothalamus, Neurosecretory system

Plaques motrices, v. Muskelfasern 
Poids de diverses parties du corps, v. Körperabschnitte

Portio uteri, v. Pflasterepithel

Potentials; action potentials, v. Nervenfasern

Potto (= Halbaffe), v. Perodicticus potto

Premature cranial synostosis in man, pathogenesis 351

Pression artérielle, v. Carotid body

Projektion des Nucleus anterior thalami, zu deren Frage 335

Prosimíae, v. Scrotalhaut

Pulpa dentis, v. Rodent teeth

Register rerum 395

Rabbit, v. Racines dentaires

Racines dentaires; leur développement; leur résorption au niveau des dents

210

163125106

252163

temporaires

Radix dentis, v. Racines dentaires

Rat; blood circulation in the rodent teeth of the rat

- ; development of the interstitial cells in the testis of the albino rat . .

$-;$ development of the rat vagina

-; das histologische Querschnittsbild des Rattenzwerchfells bei Fixierung

in verschiedenen $\Gamma$ unktionszuständen

-; v. Neurosecretory system, Ossification

Reptílía, v. Lizards

Rhachitis; antirhachitic substances, v. Epiphysial complex

Rodent teeth of the rat, blood circulation

Roots of the temporary teeth, v. Racines dentaires Rump weights, relative, v. Körperabschnitte

Säugetiere, v. Carotid body

Saison, v. Heteropneustes

Schädelbasis, v. Cranial synostosis

Schneidezahn (Ratte), v. Teeth

Scrotalhaut; Differenzierungen der Scrotalhaut von Perodíctícuspotto (Müller

1766) im Vergleich mit anderen Prosímíae 80

Seasonal changes in the ovary, v. Ovarium

Sexualverhalten, v. Scrotalhaut

Siluroídea, v. Heteropneustes

Silver impregnation, v. Pflasterepithel

Sinus caroticus, v. Carotid body

Sinus sanguins, v. Carotid body

Skelet, v. Latimería chalumnae

Skeletmuskel, v. Zwerchfell

Skeletmuskeln; Länge und Anordnung der Muskelfasern in menschlichen

Skeletmuskeln 217

Skin; glands of scrotal skin of Prosimiae, v. Scrotalhaut

Skull base, v. Cranial synostosis

Soutien, formation de soutien, v. Latímería chalumnae 
Spawning (prespawning period), v. Heteropneustes

Squelette, v. Latímería chalumnae

Staining, v. Neurosecretory system

Statistische Auswertung, v. Neurosecretory system

Stützgewebe, v. Latímería chalumnae

Subkutane Halsvenen, v. Halsvenen

Submicroscopic structure, v. Ossification, Pflasterepithel

Sustentacular tissue, v. Latímería chalumnae

Synchronisation (Gesamtsynchronisation) der Erregungsleitung, v. Nerven-

fasern

Synostosis; pathogenesis of premature cranial synostosis in man

351

Teeth; rodent teeth of the rat, blood circulation 163

- ; v. Dentes

Teratologie, v. Cranial synostosis

Territorialverhalten, v. Scrotalhaut

396 Register rerum

Testis; development of the interstitial cells in the testis of the albino rat . 125

«Tetanussubstrat, muskuläres», v. Zwerchfell

Thalamus; zur Frage der Projektion des Nucleus anterior thalami .... 335

-; v. Geniculate body

Tonofilamente, v. Pflasterepithel

«Tonussubstrat, muskuläres», v. Zwerchfell

Tooth, v. Teeth

Trunci lymphatici lumbales, v. Canal thoracique

Tuber cinereum, v. Hypothalamus

Ultrastructure, v. Ossification, Pflasterepithel Uterus (Portio vaginalis uteri), v. Pflasterepithel

106

Vagina; development of the rat vagina

Vagina, v. Pflasterepithel

Vaisseaux, v. Carotid body, Rodent teeth, Vascular supply, Venen

Vaisseaux lymphatiques, v. Canal thoracique

Variations, v. Canal thoracique, Venen

Vasa lymphatica, v. Canal thoracique

232

Vascular supply to the dental lamina during early development

Vascular supply, v. Rodent teeth

Veines, v. Venen

Vena facialis communis, v. Venen

Vena jugularis superficialis dorsalis, v. Venen

Venen; Beitrag zum Studium von Varietäten der subkutanen Halsvenen des

298

Menschen

Verkalkung, v. Ossification

Vertel-rata; the carotid body system of the higher vertebrates 265

Vessels, v. Carotid body, Rodent teeth, Vascular supply, Venen Vessels, lymph vessels, v. Canal thoracique Vitamin D, v. Epiphysial complex Vögel, v. Carotid body 
Weights, relative, of the primary body, v. Körperabschnitte Wels, v. Heteropneustes Zähne, v. Dentes

Zahnwurzeln, v. Racines dentaires

Zwerchfell; das histologische Querschnittsbild des Rattenzwerchfells bei Fixierung in verschiedenen Funktionszuständen 252

Book Reviews - Livres Nouveaux - Buchbesprechungen

Míllot, J. et J. Anthony: Anatomie de Latimeria chalumnae (Tome I: Sque-

lette, muscles et formation de soutien (Edition du C.N.R.S., Paris 1958) 280

Sieglbauer, F.: Lehrbuch der normalen Anatomie des Menschen (8. Aufl.)

(Urban u. Schwarzenberg, München/Berlin 1958)

192 